\title{
Health and technology revealing the vision on technological applications in contemporary healthcare
}

\author{
Magdalena Stoeva ${ }^{1}$
}

Published online: 23 January 2020

(C) IUPESM and Springer-Verlag GmbH Germany, part of Springer Nature 2020

\section{Dear Readers,}

Yet another year serving our professional community! Health and Technology marks its 10th anniversary! We have a year ahead of us to celebrate and to strengthen Health and Technology's position and contribution to our profession and to the society.

We at Health and Technology decided to open the anniversary year with a collection of papers touching two of the hottest topics in our profession - the vision on biomedical engineering [1] and the internet of medical things in e-health.

Prof. Dr. Almir Badnjević and Prof. Dr. Ranko Škrbić compiled an excellent selection of extended papers related to the International Conference on Medical and Biological Engineering (CMBEBIH 2019), entitled CMBEBIH 2019: biomedical engineering - share the vision [1].

The second part of Health and Technology's first issue for 2020 is the topical collection Internet Of Medical Things In E-Health. Health and Technology presents this collection thanks to the efforts of the guest editors Prof. Dr. Hassan Fouad Mohamed- El-Sayed and Prof. Dr.M.Hemalatha.

The topical collection on Internet of Medical Things in e-Health has a significant contribution to the effective research and development of e-health systems. The guest editors have emphasized the importance of Ehealth for improving the fundamental concepts in traditional medicine, including the diagnostics and treatment processes. Various computational and research problems have been referred in terms of improvement of the scientific environment and provoking growth.

Magdalena Stoeva

ms_stoeva@yahoo.com

1 Medical University of Plovdiv, Plovdiv, Bulgaria
This topical collection is focused on a few main streams discussing the latest developments, trends and solutions for IoT in e-Health:

- Internet of medical things and models in E-health systems

Healthcare efficiency and improvement have always been a subject of discussion among professionals from various disciplines. Singh et al. [2] and Veningston et al. [3] have published their work in this field.

- Internet of medical things related security

The wide application of technology and internet driven solutions in healthcare inevitably brings the question on security. Ambika et al. [4] and Jeevitha et al. [5] have focused on steganography as a leading method to ensure security in MIoT.

- Cardio IoT in healthcare

Cardio applications have been pointed out as a special item by the guest editors, thus outlining the importance of cardio related disease and medical technology for our society. The work of Vijayashree et al. [6] on developing a cardio disease classification targets a reduction of the future risks in patient health.

- Internet of medical things in health monitoring

Health monitoring is a subject of 4 papers included in the topical collection. Two of the papers Sadek et al. [7] and Hayek et al. [8] are focused on solutions related to particular cases, while the other two papers present a global view on monitoring and activity data as an integral part of the healthcare system - Thirugnanam et al. [9] and Saripalle [10].

- Internet of medical things in data analysis for detection/ diagnostics 
The application of IoT in medical diagnostics has been widely covered in nearly $30 \%$ of the papers within this topical collection. Image analysis and classification are leading research topics while at the same time authors cover different modalities. Renuka et al. [11] focus on analyzing data originating from diagnostic methods using ionizing radiation, while Nitta et al. [12] and Virupakshappa et al. [13] have targeted MRI image analysis. Image processing with particular focus on organspecific diagnostics, including oncological applications is the main subject in the works presented by Dandu et al. [14], Magar et al. [15], Polepaka et al. [16] and Rose et al. [17].

- Internet of medical things in data analysis for prediction/ prevention of communicable disease

Special attention is paid on practical solutions for IoT implementation in the prediction and prevention of communicable diseases. The Dengue virus outbreak control framework, presented by Pravin et al. [18] has a wide potential for practical implementation in certain regions.

- Internet of medical things in data analysis for prediction/ prevention of non-communicable disease

Readers have already demonstrated high interest in the prostate cancer prediction framework developed by Shakeel et al. [19].

- Internet of medical things application in hybrid studies $[20,21]$

Hybrid studies being one of the strongest contemporary methods for data collection and analysis have drawn the readers' attention. These methods are addressed by two Jayashree et al. [20] present a complex computational algorithm for early prediction of diabetic disease, while Baskar et al. [21] draws our attention to the MIoT in neurology.

The editorial team of this topical collection have gathered research, educational, engineering and business ideas to form a set of thought-provoking articles covering the various aspects of IoT in e-Health. This topical collection has already attracted a lot of interest in its on-line version, gaining a good number of citations.

Contemporary healthcare is driven by the technology, while at the same time healthcare demands are among the main factors to support technology growth. I trust this vital selection of papers will turn into another valuable contribution this process.

\section{Compliance with ethical standards}

Conflict of interest The authors declare that they have no conflict of interest.

\section{References}

1. Badnjević A. Health Technol. 2020. https://doi.org/10.1007/ s12553-020-00407-8.

2. Singh B, Acharjya DP. Computational intelligence techniques for efficient delivery of healthcare. Health Technol. 2019. https://doi. org/10.1007/s12553-018-00280-6.

3. Veningston K, Kadry S, Kalash HS, et al. Intelligent social network based data modeling for improving health care. Health Technol. 2019. https://doi.org/10.1007/s12553-019-00303-w.

4. Ambika, Biradar RL. Health Technol. 2019. https://doi.org/10. 1007/s12553-018-00289-x.

5. Jeevitha S, Amutha Prabha N. Effective payload and improved security using HMT Contourlet transform in medical image steganography. Health Technol. 2019. https://doi.org/10.1007/s12553018-00285-1.

6. Vijayashree J, Parveen Sultana H. Heart disease classification using hybridized Ruzzo-Tompa memetic based deep trained Neocognitron neural network. Health Technol. 2019. https://doi. org/10.1007/s12553-018-00292-2.

7. Sadek I, Demarasse A, Mokhtari M. Internet of things for sleep tracking: wearables vs. nonwearables. Health Technol. 2019. https://doi.org/10.1007/s12553-019-00318-3.

8. Hayek A, Telawi S, Börcsök J, et al. Health Technol. 2019. https:// doi.org/10.1007/s12553-019-00335-2 1, 10.

9. Thirugnanam T, Ghalib MR. A new healthcare architecture using IoV technology for continuous health monitoring system. Health Technol. 2019. https://doi.org/10.1007/s12553019-00306-7.

10. Saripalle RK. Leveraging FHIR to Integrate Activity Data with Electronic Health Record. Health Technol. 2019. https://doi.org/ 10.1007/s12553-019-00316-5.

11. Renuka S, Annadhason A. Mil based lung CT-image classification using CNN. Health Technol. 2019. https://doi.org/10.1007/s12553019-00300-z.

12. Nitta GR, Sravani T, Nitta S, et al. Dominant gray level based Kmeans algorithm for MRI images. Health Technol. 2019. https:// doi.org/10.1007/s12553-018-00293-1.

13. Virupakshappa, Basavaraj A. Health Technol. 2019;9:701. https:// doi.org/10.1007/s12553-018-00288-y.

14. Dandu JR, Thiyagarajan AP, Murugan PR, et al. Brain and pancreatic tumor segmentation using SRM and BPNN classification. Health Technol. 2019. https://doi.org/10.1007/ s12553-018-00284-2.

15. Magar SS, Sridharan B. Hybrid image compression technique using oscillation concept \& quasi fractal. Health Technol. 2019. https:// doi.org/10.1007/s12553-018-00282-4.

16. Polepaka S, Rao CS, Chandra Mohan M. IDSS-based Two stage classification of brain tumor using SVM. Health Technol. 2019. https://doi.org/10.1007/s12553-018-00290-4.

17. Rose RA, Annadhason A. GHT based automatic kidney image segmentation using modified AAM and GBDT. Health Technol. 2019. https://doi.org/10.1007/s12553-019-00297-5.

18. Pravin A, Jacob TP, Nagarajan G. An intelligent and secure healthcare framework for the prediction and prevention of Dengue virus outbreak using fog computing. Health Technol. 2019. https://doi.org/10.1007/s12553-019-00308-5.

19. Shakeel P, Manogaran G. Prostate cancer classification from prostate biomedical data using ant rough set algorithm with radial trained extreme learning neural network. Health Technol. 2018. https://doi.org/10.1007/s12553-018-0279-6.

20. Jayashree J, Ananda Kumar S. Hybrid swarm intelligent redundancy relevance (RR) with convolution trained compositional pattern neural network expert system for diagnosis of 
diabetes. Health Technol. 2019. https://doi.org/10.1007/ s12553-018-00291-3.

21. Baskar S, Dhulipala VRS, Shakeel PM, et al. Hybrid fuzzy based spearman rank correlation for cranial nerve palsy detection in MIoT environment. Health Technol. 2019. https://doi.org/10.1007/ s12553-019-00294-8.
Publisher's note Springer Nature remains neutral with regard to jurisdictional claims in published maps and institutional affiliations. 